\title{
A Case of Myocardial Bridge Affecting Multiple Coronary Arteries \\ Debjit Chatteriee*
}

Consultant Cardiologist, Queens Hospital, Burton on Trent, UK

\section{Article Info}

\section{Article Notes}

Received: April 11, 2018

Accepted: May 03, 2018

\section{*Correspondence:}

Dr. Debjit Chatterjee, Department of cardiology, Queens Hospital, Burton on Trent, Belvedere Road, DE13 ORB, UK; Telephone: 00447800538337;

E-mail: debjit.chatterjee@hotmail.com

(C) 2018 Chatterjee D. This article is distributed under the terms of the Creative Commons Attribution 4.0 International License.

\section{Key words:}

Myocardial bridge

Coronary artery disease

\section{ABSTRACT}

This is a rare coronary angiogram showing myocardial bridge affecting more than one coronary artery.

\section{Main manuscript}

Myocardial bridge is a congenital anomaly where a segment of epicardial coronary artery traverses through myocardium. This leads to mainly systolic compression of coronary artery. Myocardial bridge is often asymptomatic, though it can be associated with angina, acute coronary syndrome, arrhythmia and even sudden death. Incidence varies from 0.5 to $12 \%$ in different series of coronary angiogram ${ }^{1}$. Usually it affects the mid segment of the left anterior descending (LAD) artery. Myocardial bridge affecting more than one artery in coronary angiogram is very rare though has been reported before. I describe a case of muscle bridge affecting multiple arteries. This 37-yr-old gentleman presented with atypical chest pain and his ETT showed significant ST changes after 7 minutes of exercise of Bruce protocol. Coronary angiogram convincingly showed myocardial bridge affecting mid LAD and intermediate (ramus medianus) artery (Fig 1 and Fig 2 and the video clip). Echocardiogram showed no evidence of hypertrophic cardiomyopathy.

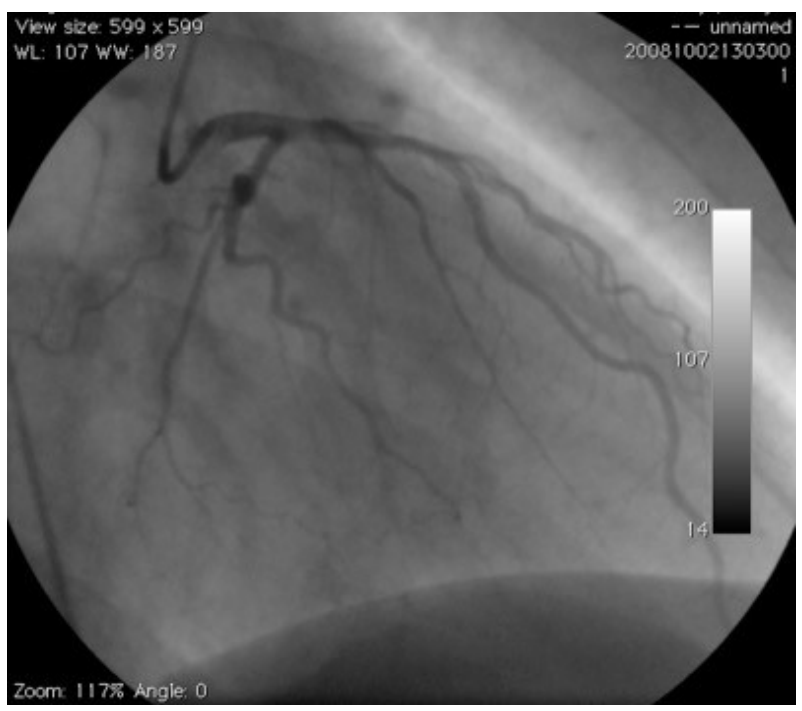

Figure 1: Coronary angiogram in diastole 


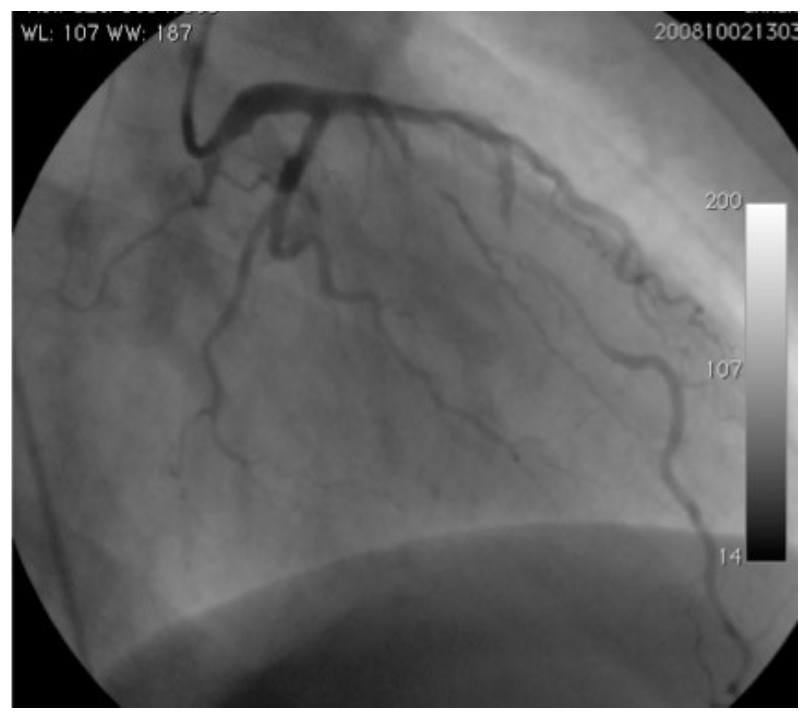

Figure 2: Coronary angiogram in systole

Majority of patients with symptomatic myocardial bridge do well in the long term. Medical treatment in the form of beta blocker should be tried to improve symptoms in the first instance. Surgical myotomy/CABG can improve symptoms in patients who have severe symptoms and failed medical therapy. Percutaneous coronary intervention should be avoided in view of recurrent revascularization reported in these patients ${ }^{2}$. The above patient has been doing well on bisoprolol $2.5 \mathrm{mg}$ od.

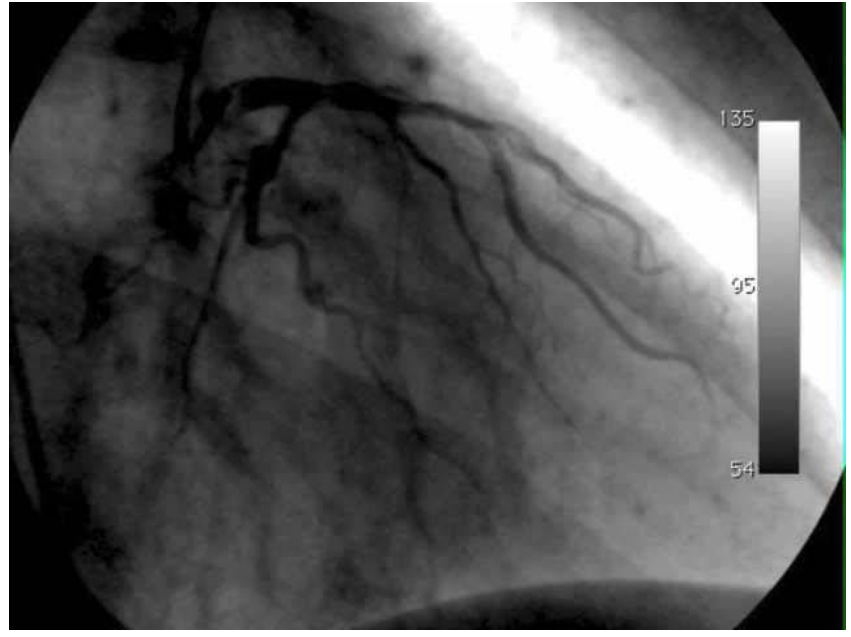

Video clip

\section{References}

1. Soran O, Pamir G, Erol C, et al. The incidence and significance of myocardial bridge in a prospectively defined population of patients undergoing coronary angiography for chest pain. Tokai J Exp Clin Med. 2000; 25: 57-60.

2. Cerrato E, Barbero U, D'Ascenzo F, et al. What is the optimal treatment for symptomatic patients with isolated coronary myocardial bridge? A systematic review and pooled analysis. J Cardiovasc Med (Hagerstown). 2017 Oct; 18(10): 758-770. 